\title{
The Sharpless Epoxidation
}

\section{Key words}

epoxidation

enantioselectivity

titanium catalysis

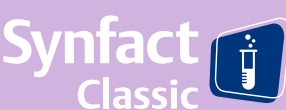

\section{Selected examples:}

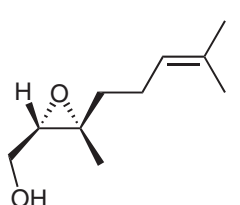

$77 \%$ yield $95 \%$ ee

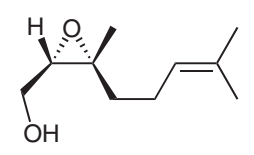

$79 \%$ yield $94 \%$ ee

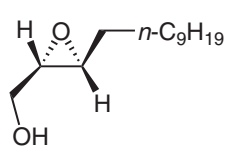

$79 \%$ yield $>95 \%$ ee

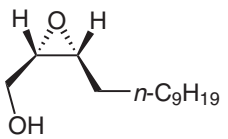

$82 \%$ yield $90 \%$ ee

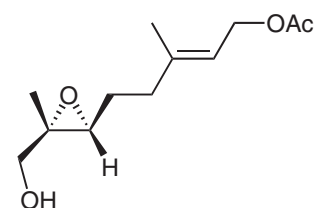

$70 \%$ yield $>95 \%$ ee

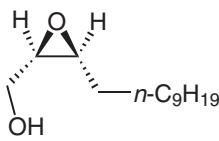

$80 \%$ yield $90 \%$ ee

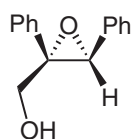

$87 \%$ yield $>95 \%$ ee

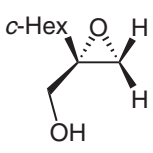

$81 \%$ yield $>95 \%$ ee
Significance: In 1980, Katsuki and Sharpless reported the asymmetric epoxidation of substituted allylic alcohols by using chiral diethyl tartrate, titanium tetraisopropoxide and tert-butyl hydroperoxide as oxidant. Given the commercial availability and low cost of the reagents, the large scope, the high enantioselectivity of the reaction, and the versatility of the resulting chiral epoxides, this method is an essential tool in organic synthesis.
Comment: Remarkably, depending on the given tartrate enantiomer, the epoxide oxygen delivery can be controlled regardless of the substitution pattern of the alkene. The naturally occurring L-(+)diethyl tartrate favored attack form the re side, whereas D-(-)-diethyl tartrate facilitated si side attack. A few years later, the involved catalyst was identified as a titanium tartrate dimer complex (for details see: M. G. Finn, K. B. Sharpless J. Am. Chem. Soc. 1991, 113, 113). 